\title{
A Cone Jet-Finding Algorithm for Heavy-Ion Collisions at LHC Energies
}

\author{
S-L Blyth ${ }^{1,2}$, M J Horner ${ }^{1,2}$, T Awes ${ }^{3}$, T Cormier ${ }^{4}$, H Gray ${ }^{1,2}$, J \\ L Klay ${ }^{5}$, S R Klein ${ }^{1}$, M van Leeuwen ${ }^{1}$, A Morsch ${ }^{6}$, G Odyniec ${ }^{1}$ \\ and A Pavlinov ${ }^{4}$ \\ ${ }^{1}$ Lawrence Berkeley National Laboratory, Berkeley, California 94720, USA \\ ${ }^{2}$ UCT-CERN Research Centre, University of Cape Town, Rondebosch 7700, South \\ Africa \\ ${ }^{3}$ Oak Ridge National Laboratory, Oak Ridge, Tennessee 37831, USA \\ ${ }^{4}$ Wayne State University, Detroit, Michigan 48202, USA \\ ${ }^{5}$ Lawrence Livermore National Laboratory, Livermore, California 94550, USA \\ ${ }^{6}$ CERN, CH-1211 Geneva 23, Switzerland
}

\begin{abstract}
Standard jet finding techniques used in elementary particle collisions have not been successful in the high track density of heavy-ion collisions. This paper describes a modified cone-type jet finding algorithm developed for the complex environment of heavy-ion collisions. The primary modification to the algorithm is the evaluation and subtraction of the large background energy, arising from uncorrelated soft hadrons, in each collision. A detailed analysis of the background energy and its event-by-event fluctuations has been performed on simulated data, and a method developed to estimate the background energy inside the jet cone from the measured energy outside the cone on an event-by-event basis. The algorithm has been tested using Monte-Carlo simulations of $\mathrm{Pb}+\mathrm{Pb}$ collisions at $\sqrt{s}=5.5 \mathrm{TeV}$ for the ALICE detector at the LHC. The algorithm can reconstruct jets with a transverse energy of $50 \mathrm{GeV}$ and above with an energy resolution of $\sim 30 \%$.
\end{abstract}

PACS numbers: 25.75.Nq 13.87.Fh

\section{Introduction}

Jet-finding techniques, a well-established tool for $p+p, e^{+}+e^{-}$and $e+p$ collisions [1], are not directly applicable in heavy-ion (HI) collisions due to the overwhelming combinatorial backgrounds from high multiplicity underlying events. For central $\mathrm{Pb}+\mathrm{Pb}$ collisions, nearly all 400 nucleons participate, leading to a high multiplicity of particles produced in simultaneous nucleon-nucleon collisions. In conventional jetfinding algorithms, this background energy will be swept up into the jet-cone, and strongly distort the reconstructed jet. This problem is also present, but to a much lesser extent, in $\mathrm{p}+\mathrm{p}$ collisions at the LHC due to multiple $\mathrm{p}+\mathrm{p}$ interactions as a result of the planned high luminosity, and due the high probability for multiple-parton interactions within a single $\mathrm{p}+\mathrm{p}$ interaction. 
Experience from RHIC (Relativistic Heavy-Ion Collider) has shown that high transverse momentum, $p_{T}$, phenomena are promising tools to investigate the hot and dense medium produced in heavy-ion collisions. The most striking results from the first five years of RHIC operation are centered around the observation that hadron production at high $p_{T}$ is strongly suppressed in central heavy-ion collisions at $\sqrt{s_{N N}}=200 \mathrm{GeV}$. High- $p_{T}$ hadrons are dominantly produced by the fragmentation of partons from hard scatterings in the initial state. The suppression of particle production is likely due to energy loss by these partons in the hot and dense matter created in the collision (possibly a Quark Gluon Plasma) [2, 3, 4, 5]. Reconstructing the remnants of these hard partons, jets, can serve as a probe of the produced medium.

Measurements of parton energy loss at RHIC have been limited to leading particle analysis and hadron correlations. Full jet reconstruction and measurement of jet energy has not been possible due to the large and highly fluctuating background of uncorrelated hadrons of the underlying event and the typical low jet energies. However, a striking improvement in jet reconstruction capability is expected in heavy-ion collisions at $\sqrt{s_{N N}}=5.5 \mathrm{TeV}$ at the LHC (Large Hadron Collider). An extrapolation of the charged particle rapidity density based on Super Proton Synchrotron (17 GeV) and RHIC (130 and $200 \mathrm{GeV}$ ) measurements suggests an additional factor of about 4 increase from RHIC top energy to LHC. The growth of the cross-section for hard processes is, however, much more dramatic. Fig. 1 1 shows the differential cross-section for inclusive jets within a pseudorapidity range of $|\eta|<1$ in $p+p$ collisions at RHIC and LHC energies from NLO pQCD as calculated by PYTHIA 6.2 [6] (left axis) and the expected annual yields in minimum bias $\mathrm{Au}+\mathrm{Au}$ and $\mathrm{Pb}+\mathrm{Pb}$ collisions (right axis). A substantial enhancement in the jet cross-section is already seen at relatively low $p_{T}(\sim 20 \mathrm{GeV})$. The kinematic reach for jet measurements is thus much larger at LHC than at RHIC, allowing the reconstruction of high energy jets above the uncorrelated background on an event-byevent basis.

Theoretical studies of partonic energy loss in a quark-gluon plasma predict that jets with intermediate transverse energies $\left(50 \mathrm{GeV} \lesssim E_{T} \lesssim 100 \mathrm{GeV}\right)$ may provide the best probe of the highly excited nuclear medium [7, 8, 9]. Partons in this energy range are expected to suffer the greatest relative energy loss and should therefore be more useful for characterizing the properties of the medium than those at asymptotically high energies. Moreover, intermediate energy jets at LHC are closer to the highest energies probed at RHIC, making direct comparisons feasible. Thus, the emphasis of this analysis is on the reconstruction of jets in the transverse energy range from 50 to $100 \mathrm{GeV}$.

A first attempt to identify and reconstruct jets on an event-by-event basis in large background $\mathrm{Pb}+\mathrm{Pb}$ collisions at the top LHC energy $(5.5 \mathrm{TeV})$ is presented in this paper. A cone-type jet-finding algorithm has been adapted from the UA1 experiment [10] and further developed to account for the heavy-ion background. The choice of various algorithm parameters is discussed and their influence on the jet energy resolution is discussed. Two different methods for determining the large fluctuating background, the 


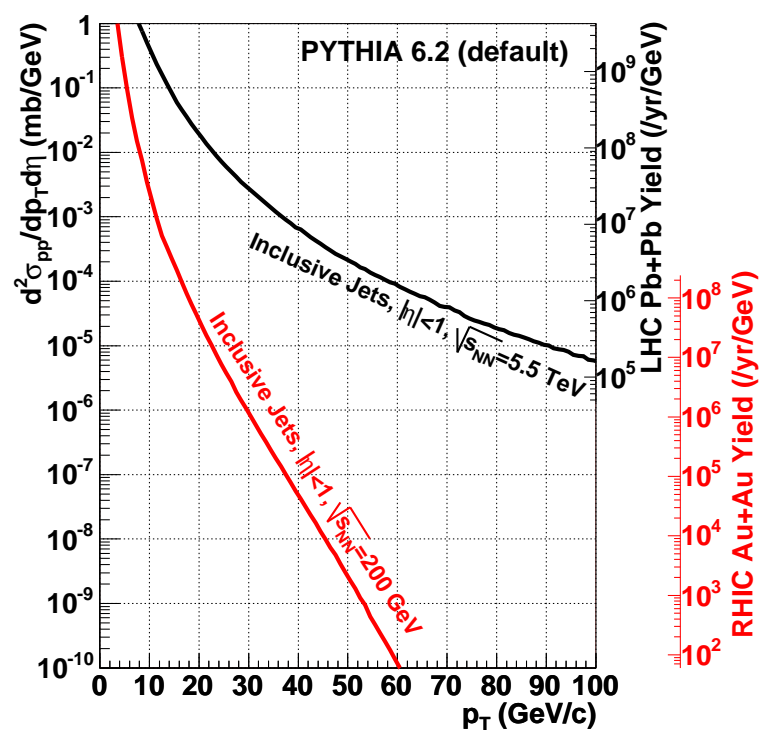

Figure 1. Differential cross-section for inclusive jets within $|\eta|<1$ in $p+p$ collisions at RHIC and LHC from default PYTHIA 6.2 (left axis). The annual yields shown on the right axes are for minimum bias $\mathrm{Pb}+\mathrm{Pb}(\mathrm{Au}+\mathrm{Au})$ collisions assuming $10^{6} \mathrm{~s}\left(10^{7}\right.$ s) running time and $0.5 \mathrm{mb}^{-1} \mathrm{~s}^{-1}\left(5.0 \mathrm{mb}^{-1} \mathrm{~s}^{-1}\right)$ luminosity at LHC (RHIC).

most challenging problem for jet-finding, are evaluated.

The presented simulations are based on the ALICE detector, using charged particle tracking for hadrons and an electromagnetic calorimeter for photon and electron detection. It has been shown previously that jets can be accurately reconstructed using this combination of detectors in $e^{+}+e^{-}$collisions at LEP (Large Electron Positron Collider) [12] and $p+\bar{p}$ collisions at the Tevatron [13]. This paper demonstrates that it can also be successful in the analysis of more complex heavy-ion collisions.

\section{The Heavy-Ion Jet Algorithm (HIJA) and Simulations}

\subsection{Description of HIJA}

This approach is based on a cone-type algorithm, developed by the UA1 collaboration [10, where the jet is defined as a group of particles in a cone of fixed radius in azimuth- $(\phi)$ and pseudorapidity- $(\eta)$ space. The algorithm refinements evaluated by the Tevatron Run II Jet Physics Group [14], such as seedless cones, splitting/merging corrections and $k_{T}$ algorithms [15], are not considered here but their feasibility could also be investigated for heavy-ion collisions.

The input to the algorithm is an energy grid in $(\eta, \phi)$ filled by a combination of transverse energy $\left(E_{T}^{\text {Cell }}=E \sin \theta_{T}\right.$, where $E$ is the total energy of the calorimeter cell and $\theta_{T}$ is the polar angle of the cell) measured by the electromagnetic calorimeter and charged track transverse momentum $\left(p_{T}\right)$ information from the tracking system. The grid covers the same fiducial area as the calorimeter, and each grid cell corresponds in 
size and position to a calorimeter cell $(\eta \times \phi=0.014 \times 0.014$ in ALICE [19]). In order to reduce the contributions from uncorrelated background particles, only charged hadrons with $p_{T}$ above a threshold $p_{T}^{\text {cut }}$ were used in the analysis.

Neutral energy is measured only in the calorimeter while charged hadronic energy is registered in both the tracking detectors and in the calorimeter. To correct for the double counting of hadronic energy, the estimated energy deposited by charged hadrons in the calorimeter is subtracted on a track-by-track basis using a parameterization of the average simulated energy deposition of charged pions in the calorimeter as a function of $\eta$ and $p_{T},\left\langle E_{H C}\left(\eta, p_{T}\right)\right\rangle$.

The algorithm consists of the following steps:

(i) Initialize the estimated background level per grid cell $\hat{E}_{T}^{B G}$ to the average over all grid cells.

(ii) Sort cells in decreasing cell energy, $E_{T}^{i}$.

(iii) For at least 2 iterations, and until the change in $\hat{E}_{T}^{B G}$ between most recent successive iterations is smaller than a set threshold

(a) Clear the jets list

(b) Flag all cells as outside a jet

(c) Execute the jet-finding loop for each cell, starting with the largest:

1. If $E_{T}^{i}-\hat{E}_{T}^{B G}>E_{T}^{\text {seed }}$, where $E_{T}^{\text {seed }}$ is a chosen threshold cell energy, and the cell is flagged as not in a jet, treat it as a jet seed candidate:

(A) Set jet centroid $\left(\eta^{C}, \phi^{C}\right)$ to the co-ordinates of the jet seed cell $\eta_{i}, \phi_{i}$.

(B) Using all cells within $\sqrt{\left(\eta^{i}-\eta^{C}\right)^{2}+\left(\phi^{i}-\phi^{C}\right)^{2}}<R$ of the initial centroid, calculate the new energy-weighted $\left(E_{T}^{i}-\hat{E}_{T}^{B G}\right)$ centroid. Set the new energy-weighted centroid to be the new initial centroid. Repeat centroid calculation iterations until the centroid does not shift by more than one cell in subsequent iterations.

(C) Store centroid as jet candidate and flag all cells within $R$ of centroid as inside a jet.

(d) Re-calculate the estimated background energy $\hat{E}_{T}^{B G}$ using the calculation described in Section 3.1

(e) For each jet candidate, calculate the energy by summing the energies of the cells in the cone and subtracting the background. If the jet energy is greater than $E_{T}^{\text {cone }}$, the minimum allowed cone energy, a jet is found.

The main algorithm parameters and their purposes are listed in Table 1.

\subsection{Description of Detector and Simulated Events}

Simulations of $\mathrm{Pb}+\mathrm{Pb}$ collisions were performed for the ALICE experimental set-up using the ALICE software framework, AliRoot [16].

The ALICE tracking detector response was approximated using a gaussian smearing (with $\sigma=1 \%$ ) of the track momentum $p$ and a conservative tracking efficiency of 


\begin{tabular}{c|l}
\hline Parameter & Description \\
\hline$E_{T}^{\text {seed }}$ & Minimum jet seed energy (after background subtraction) \\
$R$ & Radius of jet cone \\
$E_{T}^{\text {cone }}$ & Minimum jet cone energy (after background subtraction) \\
$p_{T}^{\text {cut }}$ & Minimum track $p_{T}$ \\
\hline
\end{tabular}

Table 1. Main parameters used in HIJA.

90\% (the presently anticipated ALICE tracking efficiency is 98\% [18]). The ALICE electromagnetic calorimeter $\ddagger$, a sampling calorimeter composed of 25 layers of $5 \mathrm{~mm} \times$ $5 \mathrm{~mm}$ Pb-scintillator, was simulated using GEANT 3.21. The intrinsic energy resolution for photons with energies from 25 to $200 \mathrm{GeV}$ for this device was estimated from simulation to be $\sigma(E) / E \sim 15 \% / \sqrt{E}$ [19]. The calorimeter was simulated with a fiducial acceptance of $|\eta|<0.7, \pi / 3<\phi<\pi$ and a granularity of 13824 cells $(96(\eta) \times 144(\phi))$.

A sample of heavy-ion events with calibrated high energy jets was constructed by combining the output from two Monte Carlo event generators. Jet events were generated using PYTHIA 6.2 [ 6 and these were combined with high-multiplicity $\mathrm{Pb}+\mathrm{Pb}$ 'background' events generated by HIJING 1.36 [17]. To define the input jet energy scale in PYTHIA events, the PYCELL algorithm $\S$ was used.

Calibration samples of $E_{T}=50$ and $100 \mathrm{GeV}( \pm 5 \mathrm{GeV})$ jets were generated using PYTHIA. The jet directions were restricted in pseudorapidity $(|\eta|<0.3)$ and in azimuthal angle (more than 0.26 radians from the edge of the calorimeter) to reduce the effect of the acceptance edges on the energy reconstruction. The jet energy and direction selection criteria were based on the output from PYCELL. The background event sample was composed of central HIJING events (impact parameter $\| b<5 \mathrm{fm}$ for the $10 \%$ most central collisions). The HIJING parameters were tuned for LHC energies according to 20. The charged particle rapidity density in these events is approximately 4000 at mid-rapidity. This is likely to be an overestimate of the uncorrelated background [21] at LHC.

\section{Background Energy Estimation and Choice of Algorithm Parameters}

Parton energy loss effects are expected to be most visible in jets with $E_{T} \lesssim 100 \mathrm{GeV}$ 7. 8, 9]. The algorithm parameters were chosen to optimize the energy resolution for jets with $E_{T}=50 \mathrm{GeV}$.

$\ddagger$ The final design which is still under evaluation. However, the changes under consideration are unlikely to affect jet reconstruction.

$\S$ PYCELL is the internal PYTHIA cone algorithm with $R=1$ which uses all simulated particles to reconstruct jets.

\|I In heavy-ion collisions, the impact parameter $b$ is defined as the distance of closest approach between the centres of the colliding nuclei. The most central collisions have the smallest impact parameter. 


\subsection{Background energy estimation}

In this section simulated $p+p$ and $\mathrm{Pb}+\mathrm{Pb}$ events are used to compare reconstructed jet energies to the background level and optimise the background estimation.

The left panel of Fig. 2 shows a comparison of the jet energy and RMS for $50 \mathrm{GeV}$ (triangles) and $100 \mathrm{GeV}$ jets (squares) in $p+p$ events and the total background energy (circles) from uncorrelated particle production in $\mathrm{Pb}+\mathrm{Pb}$ events, as a function of cone radius $R$. All points include a $p_{T^{-}}$cut of $2 \mathrm{GeV} / \mathrm{c}$ on charged tracks which rejects most of the background from charged particles ( $98 \%$ on average in central HIJING events). While the measured jet energy only increases for small cone radii, up to $R \sim 0.3$, the background energy increases quadratically with $R$, exceeding $100 \mathrm{GeV}$ at $R \sim 0.4$. However, it is not the magnitude of the background energy, but rather the event-byevent fluctuations (represented by the vertical bars on the circle symbols in Fig. 2) which provide the challenge to jet reconstruction and energy resolution.
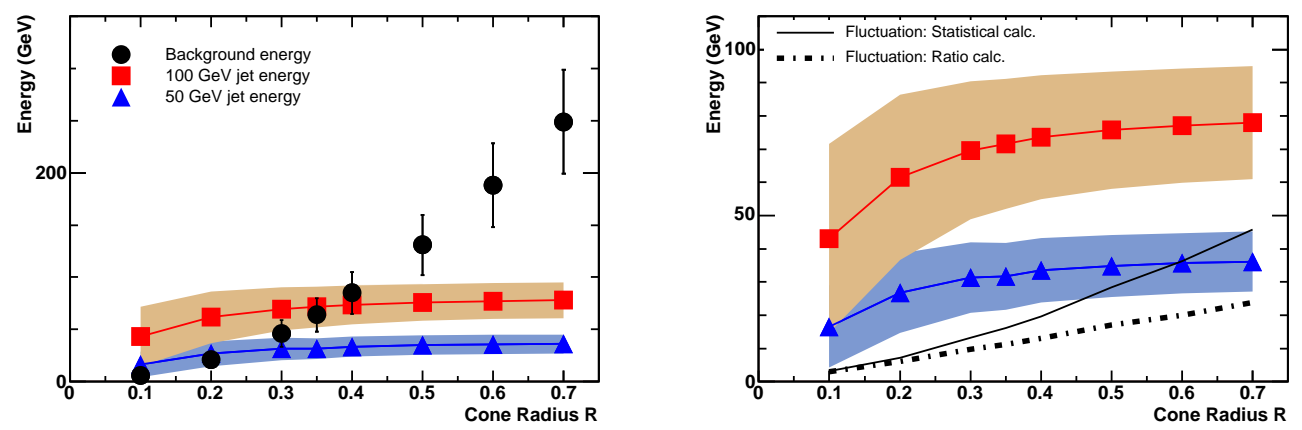

Figure 2. Left panel: Mean and RMS of background energy from central $\mathrm{Pb}+\mathrm{Pb}$ background events (circles) compared to jet energy (simulated) from $p+p$ events for $50 \mathrm{GeV}$ (triangles) and $100 \mathrm{GeV}$ (squares) jets within jet cones of varying size $R$. The shaded bands around the jet energy symbols and vertical bars on the background symbols represent the RMS values of the distributions. Right panel: Jet energy within the cone for varying cone sizes (triangles: $50 \mathrm{GeV}$ jets, squares: $100 \mathrm{GeV}$ jets) compared to the central $\mathrm{Pb}+\mathrm{Pb}$ background energy RMS in the cone calculated using two methods (solid line: statistical, dot-dashed line: ratio method). The bands represent the RMS of the jet energy distributions.

The energy resolution for jets in heavy-ion events consists of two components: the intrinsic energy resolution that would be achieved without backgrounds and a contribution from the fluctuations of the background energy contribution in the jet cone. The relative energy resolution $\sigma\left(E_{T}\right) / E_{T}$ for full jet reconstruction in the presence of backgrounds can be estimated as the quadratic sum of the jet energy resolution $\sigma\left(E_{T}^{j e t}\right)$ in $p+p$ collisions and the fluctuations of the background energy, $E_{T}^{B G}$, around the estimated background $\hat{E}_{T}^{B G}$ :

$$
\sigma\left(E_{T}\right) / E_{T} \lesssim \frac{1}{E_{T}} \sqrt{\sigma\left(E_{T}^{j e t}\right)^{2}+\sigma\left(E_{T}^{B G}-\hat{E}_{T}^{B G}\right)^{2}}
$$

The right panel of Fig. 2] shows again the jet energy for $50 \mathrm{GeV}$ (triangle markers) 
and $100 \mathrm{GeV}$ jets (square markers). The solid line shows the RMS of the background energy (vertical bars in left panel) from central $\mathrm{Pb}+\mathrm{Pb}$ events and thus indicates the constribution of background fluctuations to the jet energy resolution when using a simple average to estimate the level of background in the cone.

One of the sources of background energy fluctuations is fluctuations in the impact parameter of the collisions. The contribution of impact parameter fluctuations to the final jet energy resolution can be suppressed by estimating the energy from uncorrelated particles on an event-by-event basis from the total energy deposited outside the jet cone. For maximum statistical precision, we used the average total $E_{T}$ per cell from the entire area of the jet-finding grid outside the cone, without applying the $p_{T}$-cut on charged tracks, $\left\langle E_{T}^{\text {cell,nocut }}\right\rangle$, as the basis for the background energy estimate. The actual background energy inside the jet-cone, with cuts, is then estimated by multiplying $\left\langle E_{T}^{\text {cell,nocut }}\right\rangle$ by an average correction factor $F$ to account for the effect of the $p_{T}$-cut. The factor $F$ is calculated as the ratio of the average cell-energy in the jet-finding grid with cuts to the case without cuts, averaged over the entire sample of background events. When the analysis is applied to experimental data, $F$ can be calculated from events without detectable jets.

The resulting fluctuations of the true background energy (from HIJING $\mathrm{Pb}+\mathrm{Pb}$ events) around the estimated background energy using this procedure is indicated by the dashed line in the right panel of Fig. 2. The event-by-event estimate of the background reduces the effect of fluctuations by about a factor 2. As a result, larger cone radii can be used for jet-finding. The remaining fluctuations are dominated by fluctuations due to the finite statistical precision of the background estimate.

\subsection{Choice of parameters: cone radius}

Figure 3 shows the dependence of the relative jet energy resolution on the cone radius $R$. The relative jet energy resolution $\sigma\left(E_{T}\right) / E_{T}$ was calculated using Eq. 1 The RMS of the jet energy in $p+p$ collisions as shown in Fig. 2 was used for $\sigma\left(E_{T}^{j e t}\right)$ and the RMS of the background fluctuations (dash-dotted line in Fig. 2) was used for $\sigma\left(E_{T}^{B G}-\hat{E}_{T}^{B G}\right.$ ). For small values of $R$, the jet energy resolution improves with increasing $R$ because inand-out-of-cone fluctuations dominate (first term in Eq. 11). At larger $R$, the background fluctuations dominate and the resolution degrades with increasing $R$. For this study we chose the cone radius $(R=0.3)$ which resulted in the best energy resolution for $50 \mathrm{GeV}$ jets. This result demonstrates that for optimum jet resolution in heavy-ion collisions, the jet cone must be restricted in size, with an optimum size that decreases with decreasing jet energy. Such restrictions will be necessary to enable jet reconstruction to the lowest jet energies in central $\mathrm{Pb}+\mathrm{Pb}$ collisions. The biases introduced on the jet selection by such restrictions will require extensive systematic study, including comparisons with $\mathrm{p}+\mathrm{p}$ reference data and theoretical calculations. 


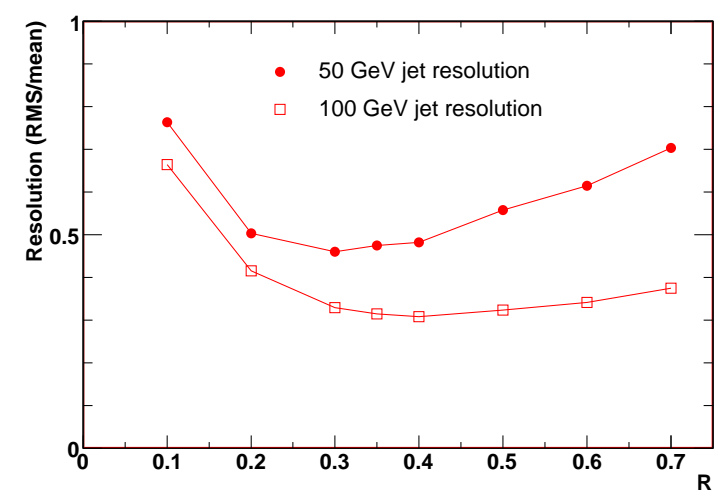

Figure 3. Energy resolution calculated as described in the text for $50 \mathrm{GeV}$ jets (circles) and $100 \mathrm{GeV}$ jets (squares) as a function of the cone radius $R$.

\subsection{Choice of parameters: seed energy and minimum jet energy}

Finding the optimal values for the jet seed energy, $E_{T}^{\text {seed }}$, and minimum accepted cone energy, $E_{T}^{\text {cone }}$, involves a trade-off between jet-finding efficiency and sample purity. To study this trade-off, we study the results of HIJA on 'parameterized $\mathrm{Pb}+\mathrm{Pb}$ ' events. These events contain only pions and kaons sampled from the $p_{T}$ and pseudorapidity distributions of the particles in HIJING events and are therefore free of jets by construction. The parameterized $\mathrm{Pb}+\mathrm{Pb}$ events had a fixed charged particle rapidity density of $d N_{c h} / d y=4000$ at mid-rapidity.

Using large values of $E_{T}^{\text {seed }}$ and/or $E_{T}^{\text {cone }}$ reduces the number of 'fake' jets (i.e. jets reconstructed by the algorithm which are not the embedded PYTHIA jet) reconstructed in the parameterized $\mathrm{Pb}+\mathrm{Pb}$ events, but also reduces the number of reconstructed jets in the event sample with embedded jets. The final parameters were selected through a trade-off between fake rate and the jet-finding efficiency for $50 \mathrm{GeV}$ jets.

\begin{tabular}{c|rl}
\hline Parameter & \multicolumn{2}{|c}{ Value } \\
\hline$E_{T}^{\text {seed }}$ & 4.6 & $\mathrm{GeV}$ \\
$R$ & 0.3 & \\
$E_{T}^{\text {cone }}$ & 14.0 & $\mathrm{GeV}$ \\
$p_{T}^{\text {cut }}$ & 2.0 & $\mathrm{GeV} / c$ \\
\hline
\end{tabular}

Table 2. Values of algorithm parameters after optimisation.

\section{Results}

This section summarizes the HIJA algorithm results for jet efficiency, energy and direction resolution in simulated $\mathrm{Pb}+\mathrm{Pb}$ collisions. 


\subsection{Jet-finding efficiency and direction resolution}

The values for $E_{T}^{\text {seed }}=4.6 \mathrm{GeV}$ and $E_{T}^{\text {cone }}=14.0 \mathrm{GeV}$ were selected, as shown in Table 2. because they resulted in (1) a high efficiency for finding $50 \mathrm{GeV}$ embedded jets in $\mathrm{Pb}+\mathrm{Pb}$ events (greater than 70\%) and (2) a low number of 'fake' jets reconstructed in simulated $\mathrm{Pb}+\mathrm{Pb}$ events (a rate of about $3 \%$ in parameterized HIJING compared to about one signal jet expected per central $\mathrm{Pb}+\mathrm{Pb}$ collision). The fraction of events accepted as containing jets for each type of event is shown in Table 3. As discussed in Sect. 3.3. parameterized HIJING events do not contain jets by construction. The true fake rate is expected to lie between the parameterized and pure HIJING values since pure hijing events do contain jets which are not able to be tagged in the simulations.

The final values of all the optimised algorithm parameters used in this study are presented in Table 2

\begin{tabular}{ccccc}
\hline & $\begin{array}{c}\text { Param. } \\
\text { HIJING }\end{array}$ & $\begin{array}{c}\text { Pure } \\
\text { HIJING }\end{array}$ & $\begin{array}{c}\mathbf{5 0 ~ G e V ~ j e t s} \\
+ \text { HIJING }\end{array}$ & $\begin{array}{c}\mathbf{1 0 0} \mathbf{G e V} \text { jets } \\
+ \text { HIJING }\end{array}$ \\
\hline Accepted & $3 \%$ & $13 \%$ & $70 \%$ & $97 \%$ \\
\hline
\end{tabular}

Table 3. Percentage of the event sample accepted by the algorithm as containing a jet using $E_{T}^{\text {seed }}=4.6 \mathrm{GeV}$ and $E_{T}^{\text {cone }}=14.0 \mathrm{GeV}$.

The high accuracy with which HIJA reconstructs jet directions is shown in Fig. 4 by the RMS values of the difference between the reconstructed and input jet directions (calculated by PYCELL), $\Delta \eta$ (triangles) and $\Delta \phi$ (circles) for $\mathrm{Pb}+\mathrm{Pb}$ (solid) and $p+p$ (open). The high background in $\mathrm{Pb}+\mathrm{Pb}$ collisions affects the direction resolution in $\eta$ and $\phi$ similarly, leading to almost equal resolutions in both directions.

For the $\mathrm{Pb}+\mathrm{Pb}$ case, the algorithm becomes more accurate with increasing jet energy because the signal to background ratio increases. For $p+p$ on the other hand, the jet direction resolution becomes slightly worse at higher jet energy. This is due to the small cone radius of $R=0.3$, which occasionally leads to two reconstructed jets instead of one. This "splitting" effect results in a small fraction of large $\Delta \eta$ and $\Delta \phi$ values, increasing with jet energy. The effect is also present in $\mathrm{Pb}+\mathrm{Pb}$, but it is offset by the background fluctuations. Possible corrections for this effect are discussed in [14].

\subsection{Jet energy resolution}

Figure 5 shows the energy distributions of reconstructed jets that have been embedded in simulated central $\mathrm{Pb}+\mathrm{Pb}$ events, using the optimized algorithm parameters (Table 2). Distributions are shown for both the raw reconstructed jet energy $E_{T}^{\text {Reco }}$ and the corrected jet energy $E_{T}^{C o r r}$. The reconstructed jet energies $E_{T}^{R e c o}$ were corrected for losses due to the small cone radius, the track $p_{T}$ cut, and missing energy from unmeasured particles by multiplication with an average correction factor $C=1 / 0.6731$, calculated from the (PYTHIA) simulations. The factor is averaged with cross-sectional weight, and therefore reproduces the actual jet energy better at low energy. The solid lines represent 


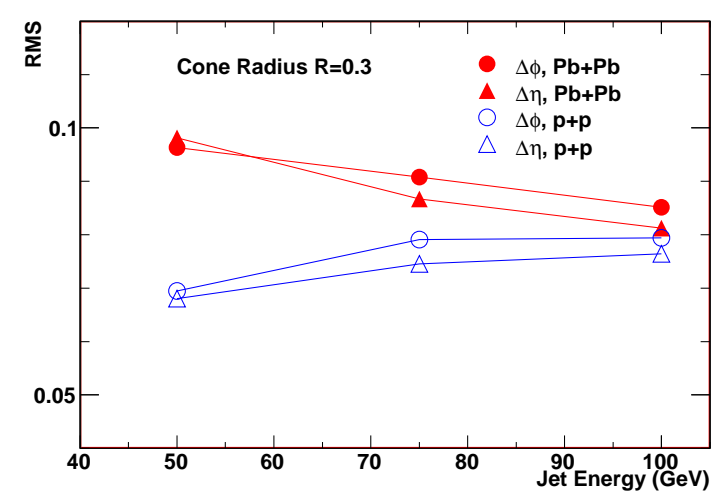

Figure 4. RMS of jet $\Delta \eta$ and $\Delta \phi$ distributions for the $\mathrm{Pb}+\mathrm{Pb}$ (solid symbols) and $p+p$ (open symbols) cases as a function of jet energy for $R=0.3$.

Gaussian fits to the corrected jet energy distributions and are used to extract the width $\sigma$ of the distributions.

The mean values and $\sigma$ of the corrected energy distributions are given in Table 4 . The mean values are within $4 \%$ of the input jet energies for all three samples.

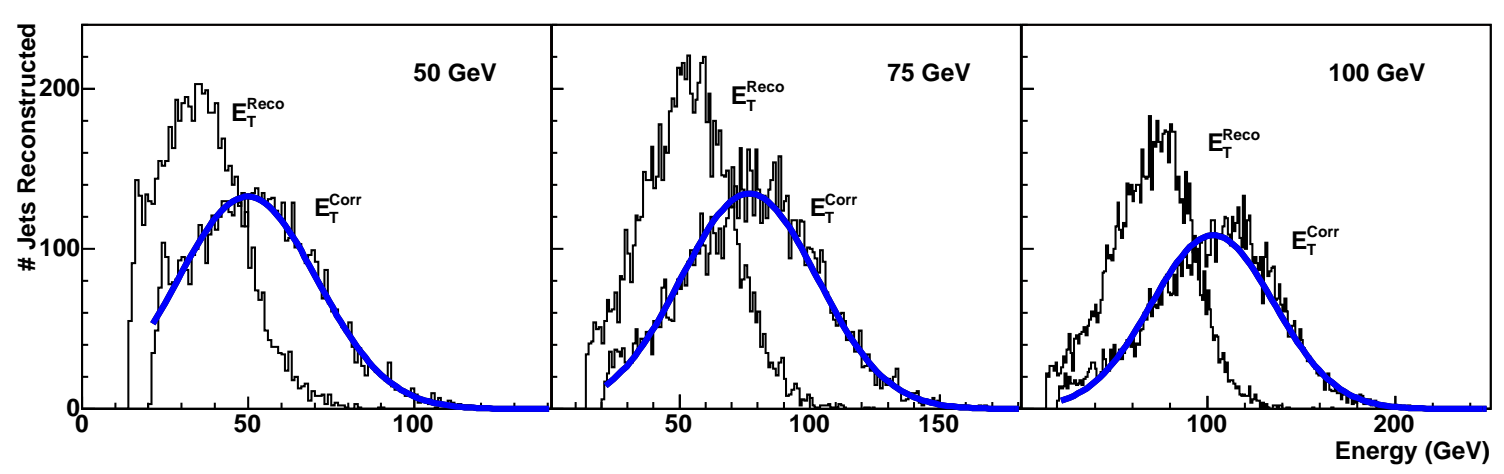

Figure 5. Reconstructed $\left(E_{T}^{\text {Reco }}\right)$ and corrected $\left(E_{T}^{C o r r}\right)$ jet energy distributions for $50 \mathrm{GeV}, 75 \mathrm{GeV}$ and $100 \mathrm{GeV}$ jets embedded in central $\mathrm{Pb}+\mathrm{Pb}$ HIJING events.

\begin{tabular}{lccc}
\hline & $\begin{array}{c}\mathbf{5 0} \mathbf{~ G e V} \text { jets } \\
+ \text { HIJING }\end{array}$ & $\begin{array}{c}\mathbf{7 5} \mathbf{~ G e V} \text { jets } \\
+ \text { HIJING }\end{array}$ & $\begin{array}{c}\mathbf{1 0 0} \mathbf{~ G e V} \text { jets } \\
+ \text { HIJING }\end{array}$ \\
\hline$\left\langle E_{T}^{\text {Reco }}\right\rangle \pm \sigma(\mathbf{G e V})$ & $34 \pm 14$ & $52 \pm 18$ & $70 \pm 22$ \\
$\left\langle E_{T}^{\text {Corr }}\right\rangle \pm \sigma(\mathbf{G e V})$ & $50 \pm 21$ & $77 \pm 26$ & $103 \pm 33$ \\
\hline
\end{tabular}

Table 4. Mean value and standard deviation $(\sigma)$ (taken from the Gaussian fits) of the reconstructed jet energy distributions (embedded in central $\mathrm{Pb}+\mathrm{Pb}$ HIJING events) for various input jet energies before and after correction for losses due to the small cone radius, the track $p_{T}$ cut, and missing energy from unmeasured particles. 


\section{Comparison to $p+p$ collision baseline}

To further separate the effects of the various algorithm cuts and the effect of background fluctuations, we have studied the effects of the algorithm cuts on $p+p$ simulations. For this purpose a cross-section weighted spectrum of PYTHIA jets with PYCELL transverse energies between 20 and $180 \mathrm{GeV}$ was generated. The influence of the HIJA cuts was examined by performing jet-finding directly on the PYTHIA particle lists without detector simulation. HIJA results for jet-finding using all particle information and cone radius $R=1.0$, agree with the reference spectrum from PYCELL.

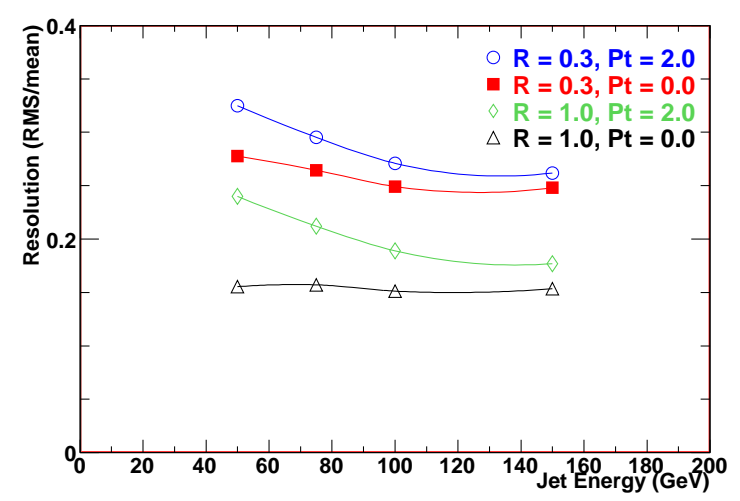

Figure 6. Energy resolution as a function of input jet energy for various HIJA cut combinations on $\mathrm{p}+\mathrm{p}$ PYTHIA events without detector simulation.

Figure 6 shows the resolutions using HIJA on the PYTHIA spectrum, as a function of energy, for two choices of $R$ and $p_{T}$-cut. To determine the energy resolution as a function of jet energy, jets were selected from the generated spectrum in a narrow $( \pm 5$ $\mathrm{GeV}$ ) energy range around the values indicated by the data points. The spread of the reconstructed energies due to the width of the selected interval was taken out by subtracting the nominal trend of reconstructed energy as a function of input energy. The resolution for the ideal case (no cuts), but excluding undetectable particles $\left(\nu, K_{L}\right.$ and neutrons) is approximately $15 \%$, independent of energy (triangle symbols). Application of the cuts on charged particle $p_{T}$ and reducing the cone radius to $R=0.3$ leads to additional loss of resolution as shown by the diamond and square markers. Using the final heavy-ion optimized parameters (see Table 2) leads to a resolution of approximately $26-35 \%$ for jets with $50 \mathrm{GeV}<E_{T}<160 \mathrm{GeV}$ (circles). This is the intrinsic limit of the jet resolution in $p+p$ using HIJA with cuts optimized for central $\mathrm{Pb}+\mathrm{Pb}$.

Figure 7 illustrates the effect of detector energy resolution and background fluctuations in $\mathrm{Pb}+\mathrm{Pb}$ events by comparing the 'ideal' jet energy resolution in $p+p$ (PYTHIA without detector simulation, open circles) with the actual jet energy resolution obtained with HIJA including all detector effects for the $p+p$ (open stars) case and detector and high multiplicity background effects for the $\mathrm{Pb}+\mathrm{Pb}$ (solid squares) case. For this figure, the $E_{T}^{\text {cone }}$ cut was applied consistently in all cases to enable a direct 


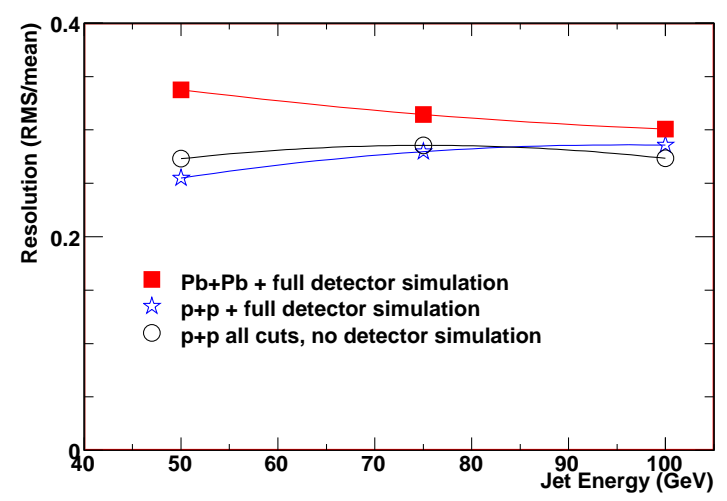

Figure 7. Reconstructed jet resolution (RMS/mean) as a function of energy for the $\mathrm{Pb}+\mathrm{Pb}$ case including detector effects (solid squares), $p+p$ case including detector effects (open stars) and $p+p$ case without detector effects (open circles).

comparison. The addition of the $E_{T}^{\text {cone }}$ cut artificially improves the resolution by a small amount compared to Figs. 3 and 6 as it truncates the distribution at low reconstructed $E_{T}$, leading to a reduced RMS value.

Detector effects (open stars) do not produce significant differences compared to the pure PYTHIA case (open circles). The small loss in jet energy resolution in $p+p$ from 50 to $100 \mathrm{GeV}$ is an artifact of the $E_{T}^{\text {cone }}$ cut. In $\mathrm{Pb}+\mathrm{Pb}$ the contribution from the fluctuating background leads to an additional spread of the reconstructed jet energy, varying from $\sim 8 \%$ for $50 \mathrm{GeV}$ jets, down to $<2 \%$ for $100 \mathrm{GeV}$ jets.

\section{Summary and Conclusions}

A UA1-based cone algorithm has been adapted to reconstruct jets in $\mathrm{Pb}+\mathrm{Pb}$ collisions at $\sqrt{s_{N N}}=5.5 \mathrm{TeV}$ at the LHC. A technique to estimate and subtract the background in heavy-ion collisions on an event-by-event basis has been developed. The contributions to the jet energy resolution from in-and-out-of-cone fluctuations, undetectable particles and the various algorithm cuts were studied. It has been shown that using this algorithm with the ALICE detectors, jets of $50 \mathrm{GeV}$ and higher transverse energies can be reconstructed on an event-by-event basis. The $p+p$ resolutions are significantly affected by the choice of parameters required to suppress the background in heavyion collisions. The final resolutions obtained with the selected algorithm parameters is $\sim 34 \%(\sim 26 \%)$ for $50 \mathrm{GeV}$ jets and $\sim 30 \%(\sim 28 \%)$ for $100 \mathrm{GeV}$ jets in $\mathrm{Pb}+\mathrm{Pb}(p+p)$ collisions. The main contribution to the degradation of the jet energy resolution in $\mathrm{Pb}+\mathrm{Pb}$ compared to $p+p$ is due to the fluctuating underlying event. This background is intrinsic to heavy-ion collisions and will be present in all experiments at the LHC. Using these techniques, the modifications to intermediate energy partons interacting in the dense nuclear medium at LHC should be experimentally accessible, providing new insights into the color structure of the quark-gluon plasma. 


\section{Acknowlegements}

SLB and HG would like to acknowledge the financial support of the National Research Foundation, South Africa. ORNL is managed by UT-Battelle, LLC, for the U.S. Department of Energy under contract DE-AC05-00OR22725. LBNL was supported by the Office of Science, Nuclear Physics, U.S. Department of Energy under Contract No. DE-AC03-76SF00098.

[1] Ellis S D and Soper D E, Phys. Rev. D 48 (1993) 3160

[2] Gyulassy M and Plumer M, Phys. Lett. B 243 (1990) 432

[3] Wang X-N, Phys. Rep. 280 (1997) 287

[4] Wang X-N and Gyulassy M, Phys. Rev. Lett. 68 (1992) 1480

[5] Wang X-N, Huang Z and Sarcevic I, Phys. Rev. Lett. 77 (1996) 231

[6] Sjostrand T et al, Computer Physics Commun. 135 (2001) 238

[7] Salgado C A and Wiedemann U A, Phys. Rev. D 68 (2003) 014008

[8] Vitev I and Gyulassy M, Phys. Rev. Lett. 89 (2002) 252301

[9] Wang Q and Wang X-N, Phys. Rev. C 71 (2005) 014903

[10] Arnison G A, et al, Phys. Lett. B 132 (1983) 214

[11] Christie W and Shestermanov K, STAR Note 196 (1995), unpublished

[12] Buskulic D, et al, Nucl. Instr. and Meth. in Physc. Res. A 360 (1995) 481

[13] Bocci A, PhD Thesis (CDF), University of Pisa (1998) unpublished

[14] Blazey G C et al, hep-ex/0005012 v2

[15] Catani A, Dokshitzer Y L, Seymour M H and Webber B R, Nucl. Phys. B 406, 187 (1993)

[16] http://aliweb.cern.ch/offline/aliroot-pro/manual.html

[17] Wang X-N and Gyulassy M, Phys. Rev. D 44 (1991) 3501

[18] ALICE TDR of the Time Projection Chamber, CERN/LHCC 2000-001

[19] ALICE-USA Collaboration, Proposal for an Electromagnetic Calorimeter for ALICE at the LHC (2003)

[20] Wang X-N, Private Communication

[21] ALICE Physics Performance Report, CERN/LHCC 2003-049 (November 2003) 